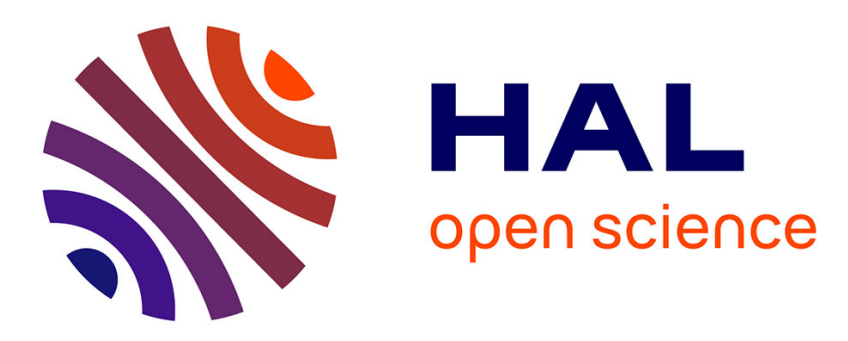

\title{
Usefulness of $\beta$-D-glucan for diagnosis and follow-up of invasive candidiasis in onco-haematological patients
}

J. Guitard, F. Isnard, M.-D. Tabone, M. Antignac, E. Brissot, Y. Senghor, A.

Petit, G. Leverger, C. Hennequin

\section{- To cite this version:}

J. Guitard, F. Isnard, M.-D. Tabone, M. Antignac, E. Brissot, et al.. Usefulness of B-D-glucan for diagnosis and follow-up of invasive candidiasis in onco-haematological patients. Journal of Infection, 2018, 76 (5), pp.483-488. 10.1016/j.jinf.2018.01.011 . hal-02297605

\section{HAL Id: hal-02297605 \\ https: / hal.sorbonne-universite.fr/hal-02297605}

Submitted on 26 Sep 2019

HAL is a multi-disciplinary open access archive for the deposit and dissemination of scientific research documents, whether they are published or not. The documents may come from teaching and research institutions in France or abroad, or from public or private research centers.
L'archive ouverte pluridisciplinaire HAL, est destinée au dépôt et à la diffusion de documents scientifiques de niveau recherche, publiés ou non, émanant des établissements d'enseignement et de recherche français ou étrangers, des laboratoires publics ou privés. 


\title{
Usefulness of $ß-D$-glucan for diagnosis and follow-up of invasive candidiasis in onco-haematological patients
}

\author{
J. Guitard a,b,* F. Isnard c , M.-D. Tabone ${ }^{\mathrm{d}}$, M. Antignac ${ }^{\mathrm{e}}$, E. Brissot ${ }^{\mathrm{c}}$, Y. Senghor ${ }^{\mathrm{a}}$, A. Petit ${ }^{\mathrm{d}}$, \\ G. Leverger ${ }^{\mathrm{d}}$, C. Hennequin ${ }^{\mathrm{a}, \mathrm{b}}$
}

\begin{abstract}
a AP-HP, Hôpital St Antoine, Service de Parasitologie-Mycologie, 34 rue Crozatier, F-75012 Paris, France
b Sorbonne Universités, UPMC Univ Paris 06, Inserm UMR S 1135, CNRS ERL 8255, Centre d'Immunologie et des Maladies Infectieuses (CIMI-Paris), 91 Bd de l'hôpital, F-75013 Paris

c AP-HP, Hôpital St Antoine, Service d'Hématologie Clinique, 34 rue Crozatier, F-75012 Paris, France

d AP-HP, Hôpital Trousseau-La Roche Guyon, Service d'Hématologie-oncologie pédiatrique, 26 av. du Docteur Arnold-Netter, 75012 Paris, France

e AP-HP, Hôpital St Antoine, Pharmacie, 34 rue Crozatier, F-75012 Paris, France
\end{abstract}

Keywords:

ß-D-glucan

Candidemia

Chronic disseminated candidiasis

Diagnosis

Outcome
A B S T R A C T

\begin{abstract}
Objectives: Definitive diagnosis of invasive candidiasis (IC) may be difficult to achieve in patients with haematological malignancy (PHM). We aimed to evaluate the performance of BDG for the diagnosis and the follow-up of IC in PHM.

Patients and methods: We retrospectively reviewed the serological data of BDG assay in adult and paediatric PHM, who developed candidemia or chronic disseminated candidiasis (CDC) through a 4-year period. Sensitivity and kinetics of BDG were determined for both clinical forms.

Results: In a panel of 3027 PHM, incidence rates of candidemia and CDC ranged between 0.74 and 0.77 and 0.30 and 0.44 according to the group of patients. At the time of diagnosis, $43.5 \%$ and $73 \%$ of cases of candidemia and CDC had a positive BDG assay, respectively. We found a significant correlation between the level of BDG at diagnosis and the outcome of candidemia ( $p=0.022$ ). In all cases of CDC, BDG negative results were obtained 2 to 6 months before recovery of the CT-scan lesions.

Conclusions: BDG exhibits a low sensitivity to detect IC in PHM, but its kinetics correlates the clinical outcome. Additional studies are warranted in patients with CDC to evaluate the interest of monitoring BDG levels to anticipate the discontinuation of antifungal maintenance therapy.
\end{abstract}

\section{Introduction}

Candida spp. remains a major cause of invasive fungal infection (IFI) in patients with haematological malignancy. ${ }^{1}$ In those patients, candidemia is the most common form, but specifically in the onco-haematological setting, some patients develop a chronic disseminated infection. ${ }^{2,3}$ Chronic disseminated candidiasis (CDC) is characterised by multiple organ involvements, mainly the liver and the spleen. The pathogenesis of CDC is thought to rely on a first candidemia episode associated with organ infectious metastases, which are further revealed when the neutropaenia resolves.

In recent studies, an incidence of candidemia between $0.5 \%$ and $1.4 \%$ and between $1.4 \%$ and $9.4 \%$ has been reported in adult and paediatric population of patients with haematological malignancy,

\footnotetext{
* Corresponding author. Laboratoire de parasitologie mycologie, Hôpital St Antoine, 34 rue Crozatier, 75012 Paris, France.

E-mail address: juliette.guitard@aphp.fr (J. Guitard).
}

respectively. ${ }^{2,4-6}$ Many case reports and some series of $\mathrm{CDC}$ have been published but, to the best of our knowledge, incidence rates are lacking for this clinical entity. ${ }^{7-9}$

The diagnosis of candidemia and CDC may be difficult. ${ }^{10}$ Candidemic patients usually present without specific clinical sign, and even fever may be inconstant. The sensitivity of blood culture is considered about $50 \%$ and may be even lower in the case of an ongoing antifungal therapy. ${ }^{11}$ The diagnosis of CDC is most often based on the demonstration of small targeted lesions in the liver and/or the spleen on a CT-scan or ultrasonography in a patient who had recently recovered from neutropaenia. A previous history of candidemia during the neutropaenia phase may have been document or not. ${ }^{12}$ Culture of biopsy specimens of infected organs is most often unsuccessful, making the diagnosis most often probable. ${ }^{10}$

The development of new diagnostic biomarkers is thus highly desirable to improve the diagnosis and guide the therapeutic management. For adults, Candida antigen (mannan) and anti-mannan antibodies assays are moderately recommended by the European Society of Clinical Microbiology and Infectious Diseases (ESCMID) and the European Conference on Infections in Leukaemia (ECIL) for the diagnosis of candidemia and $\mathrm{CDC} .{ }^{11,13}$ In paediatric population, 
those tests have not been validated, and no recommendation could be proposed by the ECIL group. ${ }^{14}$

In 2008, the revised definitions of IFI from the EORTC-MSG consensus group introduced the detection of $\beta$-D-glucan (BDG) among the biological diagnostic criteria. ${ }^{12}$ BDG is a polysaccharide component of the cell wall of the majority of pathogenic fungi. Since BDG is a panfungal marker looking specifically at the performances of the test for the diagnosis of invasive candidiasis could be difficult. Nevertheless, sensitivity between $60 \%$ and $100 \%$ for candidemia emerging in adult patients has been reported. ${ }^{15-18}$ Data in paediatric populations are scarcer. Furthermore, it has been suggested that concentration levels may be higher in healthy children leading to a higher rate of false positive. ${ }^{19}$ Nevertheless, in a previous study focused on the diagnosis of invasive fungal infections in a paediatric population with haematological malignancy, we found that the use of the threshold recommended by the manufacturer, similar for both adult and children, offers the best performance both in terms of sensitivity and specificity. ${ }^{17}$

To the best of our knowledge, none of these studies focused on the diagnosis and follow-up of CDC. In this study, we reviewed the data on BDG assay collected in patients with haematological malignancy who developed candidemia or probable CDC. We evaluated the performances of the test for the diagnosis of both clinical forms and looked at the potential interest of BDG as a surrogate marker of clinical outcome.

\section{Patients and methods}

\section{Patients}

We retrospectively reviewed all the adult and paediatric patients admitted to the haematology-oncology wards of our institution between 01/01/2013 and 31/12/2016 (4 years), diagnosed with either candidemia or CDC (see definitions below). For each patient, demographic data, type of haematological disorder and other underlying conditions were collected. Antifungal treatments received before and after the diagnosis of candidiasis were also recorded.

\section{Non-targeted antifungal management}

The standard procedures of care are detailed in Supplementary Table S1. In the paediatric department, patients presenting with a prolonged neutropaenia ( $>10$ days) associated with Candida colonisation, received antifungal prophylaxis with micafungin. In the adult ward, posaconazole was the preferred prophylactic therapy during induction phase of chemotherapy for Acute Myeloid Leukaemia and in case of graft-versus-host disease (GVHD) treated with corticosteroids. An empirical antifungal treatment consisting of liposomal amphotericin B or caspofungin was started in the case of persistence of fever 48 to 96 hours after the initiation of antibacterial therapy.

\section{Diagnosis of IFIs}

Routine procedures were applied for the diagnosis of invasive candidiasis. Multisite sampling (at least 3 among nose, rectum, armpit, and groin) for bacterial and fungal cultures was performed weekly during the neutropaenic phase. Blood cultures were performed in case of fever. Mannan antigenemia assay (Platelia Candida Antigen Plus, Biorad, France) was performed on demand, according to the manufacturer's recommendations.

During periods of neutropaenia, BDG serial assays were routinely performed once a week, according to the manufacturer's recommendations (Fungitell, Capecode, USA), on a thermostatcontrolled spectrophotometer (ELX 808, Biotek Instruments, France).
Sera were tested in duplicate, and the mean was assigned as the final result for the specimen.

\section{Candidiasis definition}

Candidemia was defined by the positivity of at least one blood culture for Candida sp. CDC was defined by the demonstration on a CT-scan or ultrasonography of small, target-like abscesses in the liver and/or spleen areas at the time of neutrophil recovery after a prolonged phase of neutropaenia. CDC was considered as probable if it occurs after an episode of candidemia within the previous month (we extend the EORTC proposed delay considering the fact that the date of imaging following the first clinical signs appeared very variable). Taking into account the ESCMID guidelines for the diagnosis and management of candidiasis, we also considered probable CDC in patients with radiological lesions associated with multisite Candida colonisation and positive biomarker(s) (mannan/ anti-mannan assay, or BDG assay). ${ }^{11}$ Finally, CDC was considered as possible if typical CT-scan or ultrasonographic lesions occur in the absence of positive biomarkers but with multisite Candida colonisation. Day 0 of the diagnosis was defined as the day of sampling of the first positive blood culture and the date of demonstration of the imaging lesions for candidemia and $C D C$, respectively.

\section{Ethical considerations}

All samples and data were collected as a part of routine diagnostic procedures. The study was retrospective, and indeed the results of the analysis did not influence the management of the patients. The study has been registered to the National Agency of Drug and Health Product Safety (ANSM) under the reference 2017A01358-45.

\section{Results}

\section{Description of the population}

Between January 2013 and December 2016, 28 episodes of candidemia, 12 probable CDC and one possible CDC were diagnosed in patients suffering from haematological malignancies. Five episodes of candidemia were excluded due to insufficient BDG data or mixed infection with another IFI (Fig. 1). Among the remaining 23 episodes, five evolved towards a probable CDC. Furthermore, seven episodes of probable CDC and one episode of possible CDC, without any previously detected candidemia, were diagnosed. Two episodes were excluded due to concomitant polyvalent immunoglobulin administration or insufficient BDG data. Thus, 10 cases of probable CDC and one possible CDC were retained for analysis (Fig. 1).

There were 12 children and 16 adult patients. The main characteristics of those patients are described in Table 1 (detailed description in Supplementary Tables S2 and S3).

The 23 episodes of fungemia were due to Candida albicans $(\mathrm{n}=7)$, Candida tropicalis $(n=6)$, Candida parapsilosis $(n=4)$, Candida kefyr $(\mathrm{n}=4)$, Candida lusitaniae $(\mathrm{n}=1)$, and Candida dubliniensis $(\mathrm{n}=1)$. Median duration of the candidemia episodes was $3.0 \pm 2.85$ days. One case was considered as persistent candidemia as positive blood cultures returned positive over a 14-day period despite catheter removal. Four episodes of candidemia were breakthrough infections. In one of these cases, a $C$. tropicalis isolate was found resistant to the antifungal used in prophylaxis, caspofungin in this case. All the 5 patients who developed CDC after candidemia received antifungals at the time of CDC diagnosis. Two among 6 patients who developed CDC without candidemia previously detected were treated with liposomal amphotericin B prophylaxis.

Incidence rates of candidemia and CDC varied between $0.74 \%$ and $0.77 \%$ and $0.30 \%$ and $0.44 \%$ according to the population of 


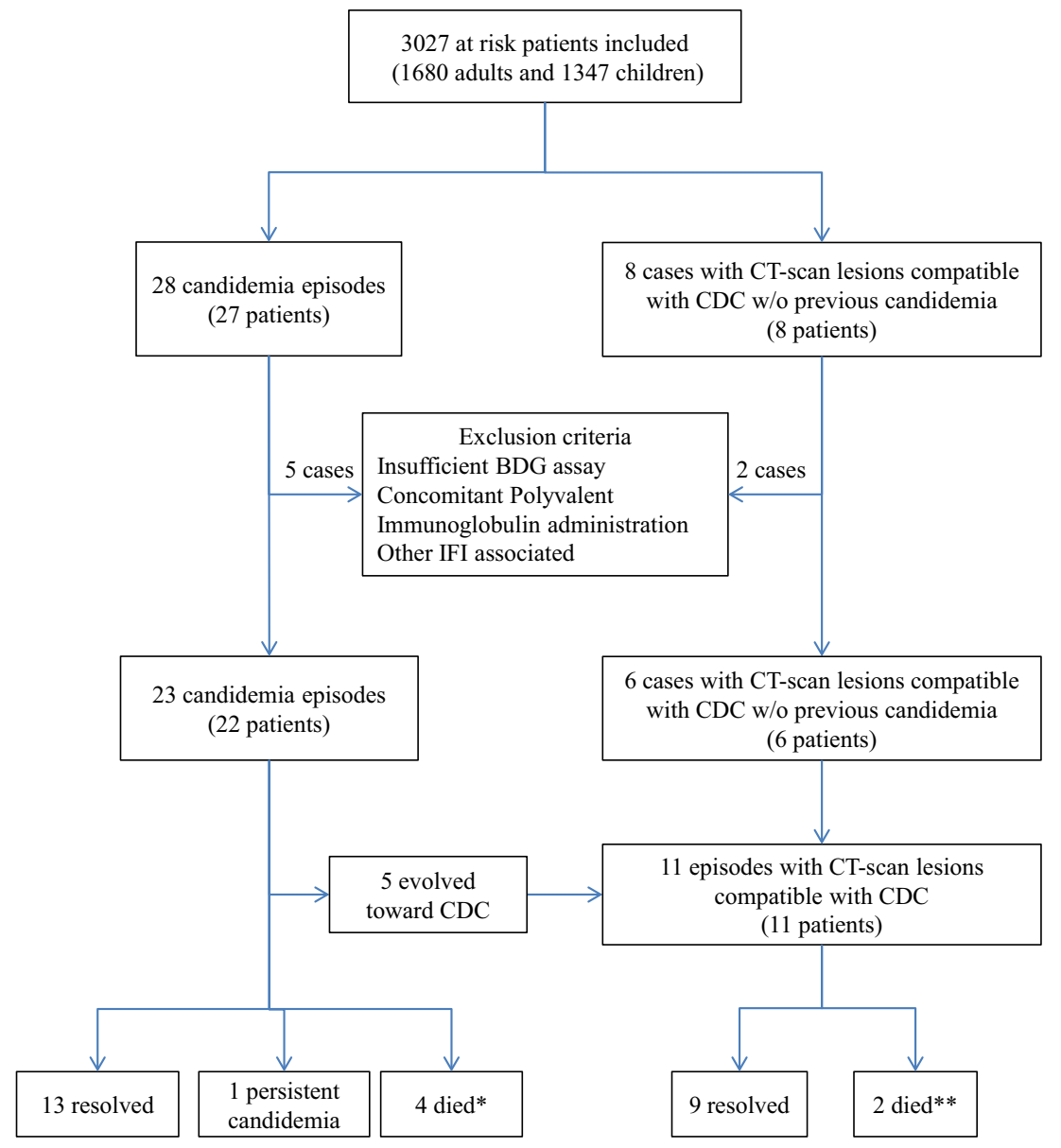

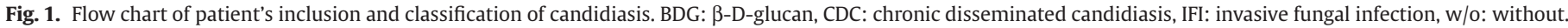
${ }^{*}$ Among which one had persistent Candida infection (positive blood cultures over a 10-day period). ${ }^{* *}$ Within 15 days. ${ }^{* * *}$ Within 3 months.

patients (Supplementary Table S4). In 5 cases, the diagnosis of CDC occurred after a median of $16 \pm 12$ days following a candidemia episode. The global mortality rate was at $17 \%$ on day 15 postdiagnosis for candidemia and $18.2 \%$ on day 90 for CDC.

\section{$\beta$-D-glucan assay performance for the diagnosis of IC and CDC}

The first BDG assay was performed with a mean delay of 1.4 days $(-17$ to +3$)$ before the first positive blood culture sampling. BDG was found positive, at the time of diagnosis or before, in 10 candidemia episodes, leading to a positivity rate of $43.5 \%$. In comparison, 8 cases out of the 14 patients (57.1\%) who benefited of a mannan assay, had a positive test at the same time. When the BDG assay was positive, the diagnosis could have been suspected before the first positive blood culture in 5 out 9 cases with available data (55\%), anticipating the diagnosis from 2 to 15 days. Among the 13 patients with a negative or equivocal BDG at the time of diagnosis, 5 (38\%) turned positive between D4 and D13 after the candidemia diagnosis.

All the 5 patients who developed CDC after candidemia had a positive BDG assay with a high level of BDG (median: $509 \mathrm{pg} / \mathrm{ml}$, range: $500-523 \mathrm{pg} / \mathrm{ml}$ ) at the time of CDC diagnosis (Supplementary Table S3). In contrast, the test was positive for 3 out of the 6 patients (50\%) presenting CDC without previous candidemia. One CDC patient with a negative BDG at the time of diagnosis turned positive 7 days later. Among the three BDG-positive patients who developed CDC without previous episode of candidemia, BDG anticipated the diagnosis by one, six, and 22 days.

\section{Potential factors leading to false negative result in $\beta$ - $D$-glucan assay}

Because the sensitivity of BDG was quite low, we looked for possible factors that may have influenced the result of the test (Supplementary Table S5 and S6). Neither Candida species (albicans vs non-albicans; $\mathrm{p}=0.67$ ), nor an ongoing antifungal treatment $(\mathrm{p}=0.64)$ impacted the result of the test. In five patients who had only positive blood cultures when drawn from the catheter, the BDG assays were negative, whereas the test was negative in 8 out of the other 18 patients $(44.4 \%)(\mathrm{p}=0.046)$.

\section{Timeline and kinetics of $\beta$-D-glucan in IC and CDC patients}

Serological follow-up of candidemia and CDC patients are described in Fig. 2. Among the 13 candidemia episodes which resolved clinically, all but 1 presented with a sharp decline in their BDG level (Fig. 2A). In contrast, among the 10 other episodes which had persistently high levels of BDG (Fig. 2B), 4 patients died, 5 evolved towards a CDC and 1 had persistent positive blood culture. We also found a correlation between the level of BDG at the time of candidemia diagnosis and the outcome at D15. Indeed, the patients who recovered had a mean level at $94 \pm 119 \mathrm{pg} / \mathrm{ml}$ whereas those who died, evolved towards CDC and those who had persistent infection had a mean level at $297 \pm 236 \mathrm{pg} / \mathrm{ml}$ (Supplementary Table S5, Student test, $\mathrm{p}=0.022$ ).

Looking at the kinetics of BDG after CDC (Fig. 2C and D), 2 patients with persistently high levels of BDG died. Regarding the 9 
Table 1

Demographics, underlying diseases and biological results of patients presenting candidiasis infection.

\begin{tabular}{|c|c|c|}
\hline & $\begin{array}{l}\text { Adult } \\
\text { patients }\end{array}$ & $\begin{array}{l}\text { Paediatric } \\
\text { patients }\end{array}$ \\
\hline Sex ratio $(F / M)$ & $9 / 7$ & $7 / 5$ \\
\hline Mean age (years $\pm \mathrm{SD}$ ) & $55.8 \pm 11.7$ & $7.9 \pm 4.2$ \\
\hline \multicolumn{3}{|l|}{ Underlying disease } \\
\hline ALL & 2 & 7 \\
\hline AML & 3 & 5 \\
\hline NHML & 3 & 0 \\
\hline Other* & 8 & 0 \\
\hline Candidemia & 13 & 10 \\
\hline CDC post-candidemia & 2 & 3 \\
\hline CDC w/o previous candidemia & 3 & 3 \\
\hline \multicolumn{3}{|c|}{ Causative species (blood cultures/colonisation) } \\
\hline C. albicans & $5 / 9$ & $2 / 6$ \\
\hline Candida non-albicans & $8 / 8$ & $8 / 6$ \\
\hline \multicolumn{3}{|c|}{ Biomarkers \% of positivity at time of diagnosis } \\
\hline (Candidemia/CDC) & $46.1 \% / 100 \%$ & $40 \% / 50 \%$ \\
\hline $\mathrm{BDG}^{\dagger}$ & $66.7 \% / 60 \%$ & $66.7 \% / 50 \%$ \\
\hline Mannan & $61.5 \% / 100 \%$ & $40 \% / 83.3 \%$ \\
\hline \multicolumn{3}{|l|}{ Mannan or BDG } \\
\hline \multicolumn{3}{|l|}{ Outcome } \\
\hline Candidemia & 7 & 6 \\
\hline Favourable outcome & $4(7.5 \pm 4.7)$ & 0 \\
\hline Death (day post-diagnosis) & 0 & 1 \\
\hline Persistent infection & 2 & 3 \\
\hline Evolution towards CDC & 3 & 6 \\
\hline $\mathrm{CDC}$ & $2(44.5 \pm 21.9)$ & 0 \\
\hline \multicolumn{3}{|l|}{ Favourable outcome } \\
\hline Death (mean day $\pm S D$ ) & & \\
\hline
\end{tabular}

" Other underlying diseases: refractory anaemia with excess blasts, myelofibrosis, multiple myeloma, Hodgkin lymphoma.

$\dagger$ Values lower than $8 \mathrm{pg} / \mathrm{ml}$ and higher than $523 \mathrm{pg} / \mathrm{ml}$ were considered as being 8 and $523 \mathrm{pg} / \mathrm{ml}$. Manufacturer's recommendations are to consider values lower than $60 \mathrm{pg} / \mathrm{ml}$ as negative, higher than $80 \mathrm{pg} / \mathrm{ml}$ as positive and equivocal between 60 and $80 \mathrm{pg} / \mathrm{ml}$.

F: female, M: male, SD: standard deviation, AML: acute myeloid leukaemia, ALL: acute lymphoid leukaemia, NHML: non Hodgkin myeloid lymphoma, BDG: $\beta$-D-glucan, CDC: chronic disseminated candidiasis, w/o: without.

patients who survived, 7 presented positive BDG during the followup, in which levels decreased within 2 to 6 months. Resolution of imaging lesions occurred 5 months to 2 years after the CDC diagnosis with remaining imaging sequel in 3 cases. In those 7 patients, BDG turned negative 2 to 14 months before a partial CT-scan recovery.

\section{Discussion}

To the best of our knowledge, our data are among the very rare to document the incidence of both candidemia and CDC in adult and paediatric patients with haematological cancer. We also show that the overall mortality rate, below $20 \%$, is lower than expected according to previous studies, $4,7,9$

Current mycological tools for the diagnosis of these infections are unsatisfactory ${ }^{10,11}$ and evaluation of a new promising biomarker such as BDG is always desirable. Nevertheless, our study has some limitations. First, because it was retrospective, BDG follow-up was missing for some patients. As candidemia became quite uncommon in those patients, we were able to analyse only 23 episodes of candidemia and 11 cases of CDC, limiting the statistical analysis. Finally, as we focused on Candida-infected patients, we could not calculate the specificity of the test in this cohort of patients. Other studies performed in onco-haematology setting had estimated this specificity between $82 \%$ and $92 \%$ in adults, ${ }^{20}$ and only $46 \%$ and $56 \%$ in children. ${ }^{17,21}$

In our study, the sensitivity of the BDG test, performed 3 days before to 3 days after the diagnosis of candidemia, was low at $43.5 \%$, in agreement with previous reports. ${ }^{15,16}$ In a prospective study focused on adult haematological patients admitted in ICU, including 8 cases of candidemia, sensitivity was found at $87.5 \%$ (for specimen drawn within 5 days before the candidemia) but with a specificity of only $35 \% .{ }^{16}$ Another prospective study reported a BDG sensitivity at $63.5 \%$ when looking at specimen sampled 1 day before to 7 days after the positive blood culture. ${ }^{15}$ Using such an interval of time in our cohort would have led to a similar sensitivity of $65 \%$. However, using such a post-diagnosis interval (7 days) clearly reduces the interest of the test for which an anticipated diagnosis is expected. In our case, the anticipation of the diagnosis was only seen for a small number of patients, $22 \%$ of candidemia and $50 \%$ of CDC without previous candidemia.

The reasons for this overall low sensitivity remain uncertain. In their study, Angebault et al and Liu et al found the sensitivity lower for non-Candida albicans species as compared to C. albicans ( $\mathrm{p}<0.01$ ), a factor not retrieved in our study. ${ }^{15,22}$ However, in our study, in 5 episodes of candidemia diagnosed with negative BDG, one can hypothesise that candidemia was only due to catheter colonisation and that this particular clinical form is not associated with the release of a significant amount of BDG in the bloodstream. Previously, we had suggested that a previous antifungal treatment, especially with echinocandin or amphotericin B, administered for more than 6 days, may be associated with negative BDG assay in a paediatric cohort. ${ }^{17}$ Again, in this larger cohort of candidemia this factor did not appear significant. Both in vitro and clinical studies are needed to decipher this issue and possibly adopt new diagnostic strategy with the BDG test.

We also looked at the potential value of the BDG in the followup of those patients. We found a correlation between the level of BDG at the time of candidemia diagnosis and the outcome at D15. This was concordant with a previous study where patients with a negative BDG at candidemia diagnosis had more chance to recover than those with a positive test at this time. ${ }^{15}$ Seric BDG levels may reflect higher persistent fungal load indicative of a poor outcome which may be either fatal or turn into CDC.

CDC diagnosis is always difficult, notably in the absence of previous candidemia, as the EORTC criteria do not include biomarkers for the classification as probable or possible CDC. ${ }^{12}$ As positive candidemia is rarely obtained in the CDC context, the ECIL and ESCMID added mannan and anti-mannan to the definition of CDC in adult patients. Furthermore, Rammaert et al also suggested adding BDG in the CDC diagnosis criteria. ${ }^{23}$ In our study, the sensitivity of blood positive culture, mannan detection, BDG detection, and the combination of these 3 criteria to detect CDC would be at $45.4 \%$, $66.9 \%, 72.7 \%$ and $90.9 \%$, respectively. Similar to candidemia, the kinetics of BDG correlated the clinical outcome of the CDC. Negativation of test occurred 2 to 6 months after the diagnosis and was associated with recovery or stabilisation of the imaging lesions. This is of significant importance since the duration of antifungal therapy is not defined for those infections and prolonged treatments expose to the risk of emergence of resistant pathogens.

In conclusion, the BDG test exhibits a low sensitivity to detect and especially anticipate the diagnosis of candidiasis. However, looking at the kinetics of the marker, the level of BDG at diagnosis can predict the outcome of those infections and thus guide the therapeutic management and future studies should determine if it can be used to discontinue the antifungal maintenance therapy.

\section{Conflict of interest}

No conflict of interest.

\section{Funding}

This research did not receive any specific grant from funding agencies in the public, commercial, or not-for-profit sectors. 

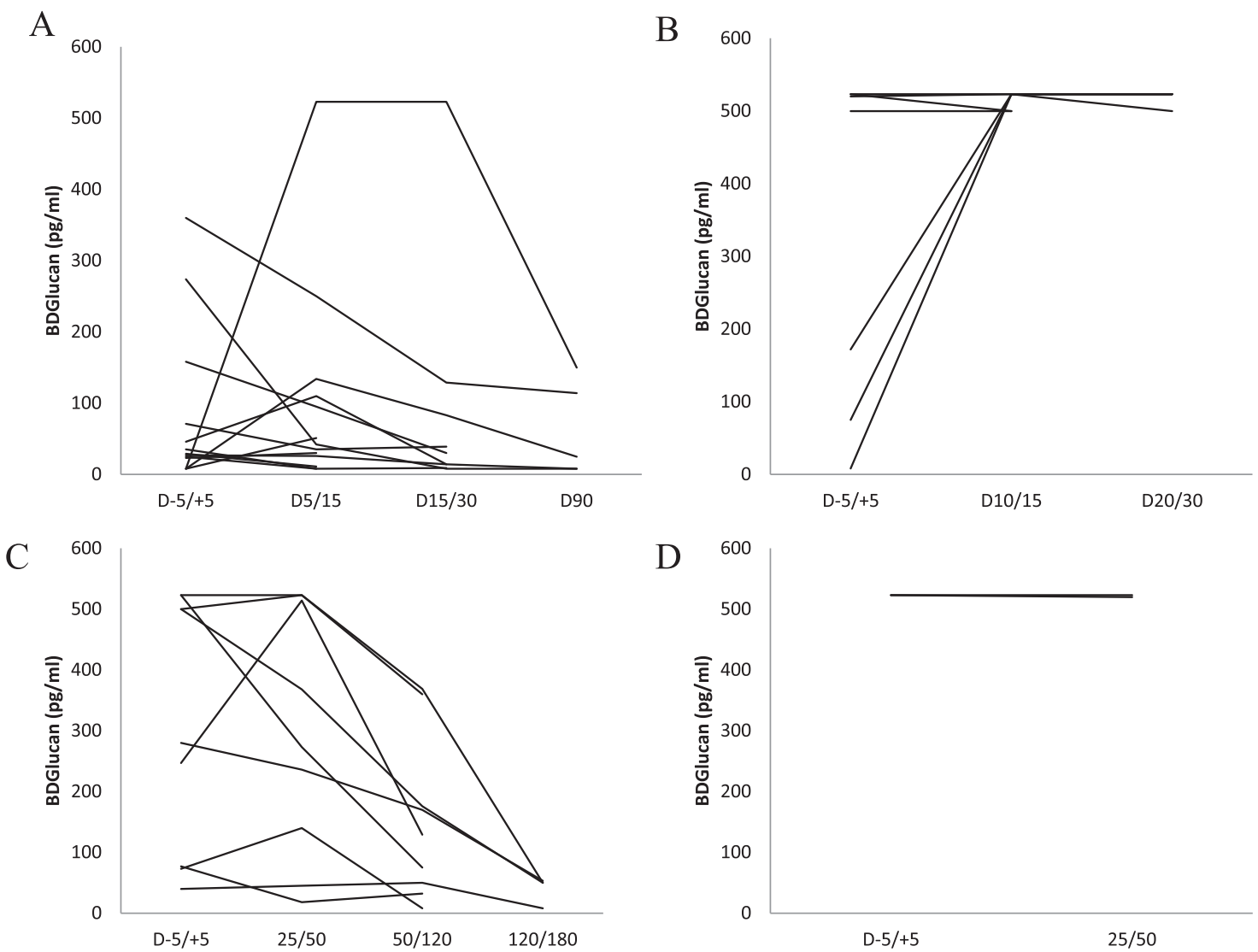

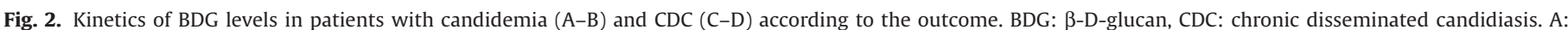

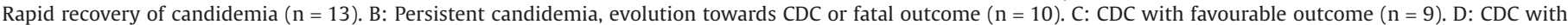
fatal outcome $(\mathrm{n}=2)$.

\section{Acknowledgements}

The authors would like to thank both the nursing team of the onco-haematological wards and the technical personnel of their lab for their excellent contribution. ${ }^{24}$

\section{Appendix A. Supplementary data}

Supplementary data related to this article can be found at https:// doi.org/10.1016/j.jinf.2018.01.011.

\section{References}

1. Neofytos D, Horn D, Anaissie E, Steinbach W, Olyaei A, Fishman J, et al. Epidemiology and outcome of invasive fungal infection in adult hematopoietic stem cell transplant recipients: analysis of Multicenter Prospective Antifungal Therapy (PATH) Alliance registry. Clin Infect Dis 2009;48:265-73. http://dx.doi.org/10 .1086/595846.

2. Montesinos P, Rodriguez-Veiga R, Boluda B, Martinez-Cuadron D, Cano I, Lancharro $\mathrm{A}$, et al. Incidence and risk factors of post-engraftment invasive fungal disease in adult allogeneic hematopoietic stem cell transplant recipients receiving oral azoles prophylaxis. Bone Marrow Transplant 2015;50:1465-72. http://dx.doi .org/10.1038/bmt.2015.181

3. Kontoyiannis DP, Marr KA, Park BJ, Alexander BD, Anaissie EJ, Walsh TJ, et al. Prospective surveillance for invasive fungal infections in hematopoietic stem cell transplant recipients, 2001-2006: overview of the Transplant-Associated Infection Surveillance Network (TRANSNET) Database. Clin Infect Dis 2010;50: 1091-100. http://dx.doi.org/10.1086/651263.

4. Gamaletsou MN, Walsh TJ, Zaoutis T, Pagoni M, Kotsopoulou M, Voulgarelis M, et al. A prospective, cohort, multicentre study of candidaemia in hospitalized adult patients with haematological malignancies. Clin Microbiol Infect 2014; 20:050-7. http://dx.doi.org/10.1111/1469-0691.12312.

5. Hale KA, Shaw PJ, Dalla-Pozza L, MacIntyre CR, Isaacs D, Sorrell TC. Epidemiology of paediatric invasive fungal infections and a case-control study of risk factors in acute leukaemia or post stem cell transplant. Br J Haematol 2010;149:26372. http://dx.doi.org/10.1111/j.1365-2141.2009.08072.x.

6. Mor M, Gilad G, Kornreich L, Fisher S, Yaniv I, Levy I. Invasive fungal infections in pediatric oncology. Pediatr Blood Cancer 2011;56:1092-7. http://dx.doi.org/ $10.1002 / \mathrm{pbc} .23005$

7. De Castro N, Mazoyer E, Porcher R, Raffoux E, Suarez F, Ribaud P, et al Hepatosplenic candidiasis in the era of new antifungal drugs: a study in Paris 2000-2007. Clin Microbiol Infect 2012;18:E185-7. http://dx.doi.org/10.1111/j 1469-0691.2012.03819.x.

8. Della Pepa R, Picardi M, Sorà F, Stamouli M, Busca A, Candoni A, et al. Successful management of chronic disseminated candidiasis in hematologic patients treated with high-dose liposomal amphotericin B: a retrospective study of the SEIFEM registry. Support Care Cancer 2016;24:3839-45. http://dx.doi.org/10 .1007/s00520-016-3208-0.

9. Pagano L, Mele L, Fianchi L, Melillo L, Martino B, D’Antonio D, et al. Chronic disseminated candidiasis in patients with hematologic malignancies. Clinical features and outcome of 29 episodes. Haematologica 2002;87:535-41.

10. Masood A, Sallah S. Chronic disseminated candidiasis in patients with acute leukemia: emphasis on diagnostic definition and treatment. Leuk Res 2005;29: 493-501. http://dx.doi.org/10.1016/j.leukres.2004.10.003.

11. Cuenca-Estrella M, Verweij PE, Arendrup MC, Arikan-Akdagli S, Bille J, Donnelly JP, et al. ESCMID* guideline for the diagnosis and management of Candida diseases 2012: diagnostic procedures. Clin Microbiol Infect 2012;18(Suppl 7):918. http://dx.doi.org/10.1111/1469-0691.12038.

12. De Pauw B, Walsh TJ, Donnelly JP, Stevens DA, Edwards JE, Calandra T, et al. Revised definitions of invasive fungal disease from the European Organization for Research and Treatment of Cancer/Invasive Fungal Infections Cooperative Group and the National Institute of Allergy and Infectious Diseases Mycoses Study Group (EORTC/MSG) Consensus Group. Clin Infect Dis 2008;46:1813-21. http:// dx.doi.org/10.1086/588660.

13. Marchetti O, Lamoth F, Mikulska M, Viscoli C, Verweij P, Bretagne S, et al. ECIL recommendations for the use of biological markers for the diagnosis of invasive fungal diseases in leukemic patients and hematopoietic SCT recipients. Bone Marrow Transplant 2012;47:846-54. http://dx.doi.org/10.1038/bmt.2011.178.

14. Groll AH, Castagnola E, Cesaro S, Dalle JH, Engelhard D, Hope W, et al. Fourth European Conference on Infections in Leukaemia (ECIL-4): guidelines for diagnosis, prevention, and treatment of invasive fungal diseases in paediatric patients with cancer or allogeneic haemopoietic stem-cell transplantation. Lancet Oncol 2014;15:e327-40. http://dx.doi.org/10.1016/s1470-2045(14)70017-8. 
15. Angebault C, Lanternier F, Dalle F, Schrimpf C, Roupie AL, Dupuis A, et al. Prospective evaluation of serum beta-glucan testing in patients with probable or proven fungal diseases. Open Forum Infect Dis 2016;3:ofw128. http://dx.doi .org/10.1093/ofid/ofw128.

16. Azoulay E, Guigue N, Darmon M, Mokart D, Lemiale V, Kouatchet A, et al. (1, 3)-beta-D-glucan assay for diagnosing invasive fungal infections in critically ill patients with hematological malignancies. Oncotarget 2016;7(16):21484-95. http://dx.doi.org/10.18632/oncotarget.7471.

17. Guitard J, Tabone MD, Senghor Y, Cros C, Moissenet D, Markowicz K, et al. Detection of beta-D-glucan for the diagnosis of invasive fungal infection in children with hematological malignancy. J Infect 2016;73:607-15. http://dx.doi.org/10 .1016/j.jinf.2016.07.007.

18. Hammarstrom H, Kondori N, Friman V, Wenneras C. How to interpret serum levels of beta-glucan for the diagnosis of invasive fungal infections in adult high-risk hematology patients: optimal cut-off levels and confounding factors. Eur J Clin Microbiol Infect Dis 2015;34:917-25. http://dx.doi.org/10.1007/s10096-014 $-2302-9$.

19. Smith PB, Benjamin DK Jr, Alexander BD, Johnson MD, Finkelman MA, Steinbach WJ. Quantification of 1,3-beta-D-glucan levels in children: preliminary data for diagnostic use of the beta-glucan assay in a pediatric setting. Clin Vaccine Immunol 2007;14:924-5. http://dx.doi.org/10.1128/cvi.00025-07.

20. Karageorgopoulos DE, Vouloumanou EK, Ntziora F, Michalopoulos A, Rafailidis PI, Falagas ME. beta-D-glucan assay for the diagnosis of invasive fungal infections: a meta-analysis. Clin Infect Dis 2011;52:750-70. http://dx.doi.org/10.1093/cid/ ciq206.

21. Badiee P, Alborzi A, Karimi M, Pourabbas B, Haddadi P, Mardaneh J, et al. Diagnostic potential of nested PCR, galactomannan EIA, and beta-D-glucan for invasive aspergillosis in pediatric patients. J Infect Dev Ctries 2012;6:352-7. http:// dx.doi.org/10.3855/jidc. 2110 .

22. Liu Y, Chen F, Zhu X, Shen L, Zhang SX. Evaluation of a novel plasma (1,3)-betad-glucan detection assay for diagnosis of candidemia in pediatric patients. J Clin Microbiol 2015;53:3017-20. http://dx.doi.org/10.1128/JCM.00673-15.

23. Rammaert B, Desjardins A, Lortholary O. New insights into hepatosplenic candidosis, a manifestation of chronic disseminated candidosis. Mycoses 2012; 55:e74-84. http://dx.doi.org/10.1111/j.1439-0507.2012.02182.x.

24. Abe M, Kimura M, Araoka H, Taniguchi S, Yoneyama A. Serum (1,3)-beta-Dglucan is an inefficient marker of breakthrough candidemia. Med Mycol 2014; 52:835-40. http://dx.doi.org/10.1093/mmy/myu066. 